\title{
Gottes Mut und Tillichs Beitrag. Zu den Be- sonderheiten und der bleibenden Relevanz von Paul Tillichs Angsttheorie
}

Sabine Joy Ihben-Bahl

Wollte sich der Theologe bzw. die Theologin mit Gefühlen oder Emotionen auseinandersetzen, kann er oder sie auf einen reichhaltigen Fundus zurückgreifen:Thomas von Aquin oder Schleiermacher etwa haben sich intensiv mit dem gefühlten Leben auseinandergesetzt. Und: Er oder sie sollte es sogar tun - es wird regelrecht gefordert, ${ }^{1}$ denn die Beachtung des gefühlten Lebens erlebt bereits seit einiger Zeit eine Renaissance, es gibt einen Aufschwung in der Emotionsforschung in allen Disziplinen und auch im Kontext gesellschaftlich-politischer Debatten werden Emotionen verstärkt thematisiert. ${ }^{2}$ Dabei wird zunehmend für die Aufwertung der Emotionen plädiert: Sie gelten schon längst nicht mehr als Phänomene, die das Leben nur begleiten oder gar stören, sondern als notwendige Instrumente, um die Welt und sich selbst darin zu erfassen, so dass auch die Gestaltung von Welt möglich wird. Die bereits vor einigen Jahrzehnten begonnene "kognitive Wende« wird spätestens dann ernst genommen, wenn Gefühlen, zumindest bestimmten, eine rationale Dimension zugestanden, ihnen z.T. axiologische Eigenschaften zuerkannt werden und ihnen letztlich zugetraut wird, dass sie evaluativ-repräsentational die Wirklichkeit wiedergeben. ${ }^{3}$ Begrifflich werden im neuen Diskurs diese Gefühle häufig als "Emotionen" bezeichnet, um sie von weniger komplexen Gefühlen zu unterschei-

\footnotetext{
1 Der neue Diskurs habe die Theologie noch nicht erreicht, behaupten Roderich Barth und Christopher Zarnow, vgl. Dies., Das Projekt einer Theologie der Gefühle, in: Dies. (Hg.), Theologie der Gefühle, Berlin/Boston 2015, 1-19, hier 1.

2 Neben den unzähligen Beiträgen zu Angst und (populistischer) Angstmacherei in der Öffentlichkeit gibt es zeitdiagnostische Analysen wie die des Soziologen und Philosophen Heinz Bude, Gesellschaft der Angst, Hamburg 2014, der Philosophin Martha Nussbaum, Königreich der Angst. Gedanken zur aktuellen politischen Krise, Darmstadt 2019 und Barry Glassner, der die Angst der US-Amerikaner beschreibt: Ders., The Culture of Fear: Why Americans Are Afraid of the Wrong Things: Crime, Drugs, Minorities, Teen Moms, Killer Kids, Mutant Microbes, Plane Crashes, Road Rage \& So Much More, Philadelphia ${ }^{2} 2010$.

3 Vgl. dazu u.a. Sabine A. Döring, Allgemeine Einleitung: Philosophie der Gefühle heute, in: Dies. (Hg.), Philosophie der Gefühle, Frankfurt am Main ${ }^{3} 2013,12-68$, bes. 17 oder Martin Hartmann, Gefühle. Wie die Wissenschaften sie erklären, Frankfurt am Main/New York ${ }^{22} 2010,15.86-88$ u.a.
} 
den. ${ }^{4}$ Zudem wird - damit notwendig verbunden - der größere Horizont des Verhältnisses von Vernunft und Gefühl und der immer noch schwelende Leib-Seele-Dualismus von Neuem bearbeitet. Wenn zunehmend betont wird, dass Gefühle in ihrer physischen und psychischen Realität der Verortung und dem Sein des Menschen in der Welt gleichermaßen dienen, muss sich die Frage stellen, wie ihr Zusammenspiel zu verstehen ist. Der Gefühlsdiskurs, der diese philosophisch-anthropologischen Grundsatzfragen mitbedenkt und damit an den der Aufklärungszeit erinnert, ${ }^{5}$ bekommt heutzutage nicht zuletzt durch neurowissenschaftliche Erkenntnisse neuen Auftrieb, durch die die Aufwertung des Gefühlten, aber auch die genannten Horizonte inzwischen auf andere Weise relevant werden. Einige Neurowissenschaftler machen ihre Ergebnisse in einer Weise verfügbar, dass ihre Forschung auch einem größeren, nicht nur wissenschaftlichen Publikum bekannt wird. ${ }^{6}$

Theolog*innen sollten sich also mit dem gefühlten Sein des Menschen auseinandersetzen. Wer sich nun dezidiert der "Angst" widmen will, findet in Paul Tillich (1886-1965) einen Gesprächspartner. Der Mut zum Sein ${ }^{7}$ oder, im Original, The Courage to $\mathrm{Be}^{8}$, Tillichs kleine Schrift zur Angst aus den 50er Jahren, war nicht nur zur Zeit ihrer Veröffentlichung ein »Verkaufsschlager und machte Tillich zu einem der populären Intellektuellen der USA, ${ }^{9}$ sondern ist nach wie vor einem großen theologischen Publikum gut bekannt.

Doch muss gefragt werden, ob es lohnenswert ist, diese Schrift noch immer zu Rate zu ziehen, wenn man sich dem Thema Angst im Kontext neuerer Forschungsergebnisse und im Horizont der Herausforderungen disziplinübergreifenden Arbeitens im gegenwärtigen Emotionsdiskurs stellen muss. Natürlich war es damals innovativ, dass Tillich sich u.a. mit dem Angstbegriff "Angst-rele-

\footnotetext{
4 Vgl. Döring, Allgemeine Einleitung, 13f.

5 Vgl. Barth/Zarnow, Das Projekt, 5-7; vgl. zur Aufklärungsdebatte selbst Wolfhart Henckmann, "Gefühl«, in: Handbuch Philosophischer Grundbegriffe, hg. von Hermann Krings/Hans M. Baumgartner/Christoph Wild, Studienausgabe, Bd. 2: Dialektik - Gesellschaft, München 1973, 520-535, hier 524.

${ }_{6}$ Gemeint sind u.a.Joseph LeDoux oder Antonio Damasio. S. Joseph LeDoux, Angst: Wie wir Furcht und Angst begreifen und therapieren können, wenn wir das Gehirn verstehen, Wals bei Salzburg 2016, Antonio R. Damasio, Der Spinoza-Effekt: Wie Gefühle unser Leben bestimmen, München 2003 u. Ders., Descartes' Irrtum: Fühlen, Denken und das menschliche Gehirn, München 1995.

7 Paul Tillich, Der Mut zum Sein (engl. 1952), übers. v. Ingeborg C. Henel, Berlin/ München/Boston ${ }^{2} 2015$.

8 Paul Tillich, The Courage to Be [1952], New Haven/London 2000.

9 Vgl. Peter J. Gomes, Introduction, in: Paul Tillich, The Courage to Be [1952], New Haven/London 2000, XI-XXXIII, hier XII. XV.
} 
vanter" Philosophen (Søren Kierkegaard, Martin Heidegger) oder post-freudianischer, amerikanischer Psychologen unterschiedlicher Couleur auseinandergesetzt hat und somit Angst zu einem theologisch relevanten Thema erklären konnte, das eine zentrale Rolle für den Menschen und seine Beziehung zum alles umgebenden "Sein-Selbst« - Tillichs Begriff für Gott - spielt, und er sich damit gegen eine Angst-relativierende oder Angst-degradierende christliche Botschaft verwehrt hat. Die Erkenntnis jedoch, dass Angst nicht aus der Glaubenserfahrung zu eliminieren ist und innerhalb des Glaubenslebens konstruktive Momente bereithält, ist heutzutage nicht mehr neu. Gleiches gilt auch von jenen "Angstzeiten", die Tillich im Mut zum Sein beschrieben und damit eine Interdependenz zwischen Epochen und der in ihnen hauptsächlich präsenten Angst behauptet hat. ${ }^{10}$ Diese Beobachtung war in den 50er Jahren durchaus bedeutsam und die Grundannahme, über die Angst eine Gesellschaft analysieren zu können, wird auch gegenwärtig geteilt. ${ }^{11}$ Doch gerade weil Tillich selbst zeitsensibel war und mit soziologischem Interesse die eigene Zeit genau beobachtet hat, muss gefragt werden, ob seine Angstbeschreibung der Neuzeit noch über unsere Angst Auskunft geben kann. Tillich schrieb sein Angstbuch nach einer für ihn traumatischen Zeit als Feldprediger im Ersten Weltkrieg und nachdem er aufgrund seiner politischen Gesinnung Deutschland 1933 verlassen und ins Exil in die USA flüchten musste. Auch war er Zeuge der atomaren Aufrüstung - hier zeigt sich viel zeitgebundenes Angstpotenzial, das nicht mehr unser Angstpotenzial ist. ${ }^{12}$

Tillichs Angstbegriff - früher modern, heute vielleicht noch interessant, doch insgesamt überholt? Keineswegs. Tillichs Überlegungen zur Angst, die von ihm disziplinübergreifend erarbeitet und zeitdiagnostisch präzisiert wurden, bilden vielmehr eine komplexe, kohärente Angsttheorie, die noch für den heutigen Angstdiskurs richtungsweisend sein kann, ja, Tillichs Angsttheorie wurde überhaupt erst $\mathrm{zu}$ einer solchen, weil sie mit seinem theologischen System in eine kohärente Wirklichkeitsbeschreibung eingebunden ist, welche auch die Erkenntnisse zur Angst unterschiedlicher Disziplinen plausibel machen und konstruktiv miteinbeziehen kann, indem er die Rolle der Disziplinen im Wissenschaftsdiskurs selbst reflektiert. ${ }^{13}$ Tillichs

\footnotetext{
${ }^{10}$ Vgl. Tillich, Mut, 39-46.

${ }^{11}$ Erneut sei verwiesen auf Bude, Gesellschaft, bes. 10.14.

${ }^{12}$ S. zur Biografie Tillichs u.a. Werner Schüssler/Erdmann Sturm, Paul Tillich. Leben - Werk - Wirkung, Darmstadt 2007, 3-25.

${ }^{13}$ Dies habe ich ausfuhrlich in meiner Dissertation zu begründen versucht, auf die sich die Ausführungen in diesem Beitrag beziehen. S. Sabine Joy Ihben-Bahl, Angst
} 
Beitrag zur Angst kann noch immer eine Vorreiterfunktion zukommen, indem sie als wirklich transdisziplinär verstanden werden kann (1.). Damit entwickelt Tillich eine Angsttheorie, die Angst in ihren unterschiedlichen Gestalten, d.h. in ihrer existentiellen "Grundform" und ihre pathologischen Ausformungen und ebenso in ihrer Wechselwirkung mit, bei gleichzeitiger und notwendiger Unterscheidung von Furcht erklären kann (2.). Der disziplinübergreifende Angstbegriff kann dennoch als wirklich theologisch verstanden werden - und das unter Zuhilfenahme der Philosophie (3.). Durch das denkrichtige System, das er entwickelt und in dem sein Angstbegriff steht, wird deutlich, dass sein Angstbegriff gut begründet und deshalb, noch heute, wirklich hilfreich ist (4.).

\section{Wirklich transdisziplinär}

In den Wissenschaften wird verstärkt disziplinübergreifend gearbeitet. Oft wird von "Transdisziplinarität" gesprochen, womit u.a. die Offenheit der Disziplinen ausgedrückt wird, sich ihre Grenzen einzugestehen, wenn sie einen Gegenstand nur in ihren eigenen disziplinären Grenzen betrachten würden. Transdisziplinäre Forscher wollen mehr als »kooperieren « ${ }^{14}$, wollen sich zugleich kritisch als zusammenarbeitende Disziplinen selbst reflektieren:

„Das verlangt eine Haltung (selbst)kritischer Reflexion über die Potenziale und Grenzen der eigenen sowie anderer Disziplin(en); es berücksichtigt deren historische Entwicklung und kulturelle Einbettung. Disziplinen werden als Orte der Orientierung, Identifikation und Ressourcen geschätzt, aber dennoch als stetig im Wandel befindlich begriffen. $\|^{15}$

Dahinter steht die Einsicht, dass komplexe Phänomene nur angemessen betrachtet werden können, wenn man sich ihnen multiperspektivisch annähert: Angst ist das beste Beispiel: Wie könnte sie, die sowohl empirisch beobachtbar, psychologisch und soziologisch abbildbar ist und zugleich Philosophie und Theologie schon immer zum Nachdenken animiert hat, die in ihren unterschiedlichen symbolischen Ausdrucksmöglichkeiten Gegenstand der Kunst war

und die eine Wirklichkeit. Paul Tillichs transdisziplinäre Angsttheorie im Dialog mit gegenwärtigen Emotionskonzepten, Tübingen 2020.

${ }^{14}$ https://www.rewi.hu-berlin.de/de/lf/ls/bae/wissen/intertransdisziplinaritaet/index.html (16.11.2019).

${ }^{15}$ Ebd. 
und ist, nur von einer Disziplin bedacht werden, wenn sie wirklich begriffen werden soll? Angst muss zwar auch Gegenstand einzelner Disziplinen bleiben, die eine Vielzahl von Erkenntnissen über sie sammeln können, doch eine Theorie der Angst kann nur transdisziplinär entwickelt werden.

Transdisziplinären Forschergruppen ist durchaus der Problemhorizont bewusst, in dem sie stehen. Dass Wissenschaften andere Wissenschaften als Wissenschaften begreifen, obwohl sie unterschiedliche "Sprachen sprechen $^{16}$ und ihnen z.T. ganz unterschiedliche Weltbilder zugrunde liegen, muss von ihnen reflektiert werden, bevor der Gegenstand selbst überhaupt in den Blick geraten darf.

Tillichs Angsttheorie ist nun auf genuine Weise das Ergebnis eines transdisziplinären Arbeitens: Der Angstbegriff aus der Spätphase seines Wirkens wäre nicht möglich ohne das System, in das er integriert ist und das sich im Laufe seiner gesamten Schaffenszeit entwickelt hat. Die »idealistische" Prägung, dezidiert die intensive Beschäftigung mit Schellings (Spät-)Philosophie, die ihm verständlich macht, dass der Idealismus nicht genügt, um Wirklichkeit zu begreifen, sondern mit einem Realismus in Wechselwirkung stehen müsse und der Natur nachzugehen sei, um dem Lebendigen gerecht zu werden, bleibt für sein eigenes System die grundlegende Wahrheit. ${ }^{17}$ In seinem System, das er in den 60er Jahren mit seiner Systematischen Theologie abschließt, ${ }^{18}$ werden die unterschiedlichen wissenschaftlichen Disziplinen verortet und bilden hierbei Perspektiven auf die eine Wirklichkeit. Die Auseinandersetzung mit den Einzeldisziplinen findet bereits in Tillichs System der Wissenschaften nach Gegenständen und Methoden ${ }^{19}$ in den 20er Jahren des 20. Jahrhunderts statt. Dort unterteilt er in Anbetracht der anhand von drei unterschiedlichen

\footnotetext{
${ }^{16} \mathrm{Zu}$ dem Problem der regelrechten "Sprachbarrieren« disziplinübergreifender Forschung vgl. Irmline Veit-Brause, Die Interdisziplinarität der Begriffsgeschichte als Brücke zwischen den Disziplinen, in: Gunter Scholz (Hg.), Die Interdisziplinarität der Begriffsgeschichte, Archiv für Begriffsgeschichte, Jg. 2000, Hamburg 2000, 15-29, hier 26.

${ }^{17}$ Vgl. Friedrich Wilhelm J. Schelling, Über das Wesen der menschlichen Freiheit [1809], Einleitung und Anmerkungen von Horst Fuhrmans, Stuttgart 2017, 60-64. 68. "Idealismus ist Seele der Philosophie; Realismus ihr Leib; nur beide zusammen machen ein lebendiges Ganzes aus."Schelling, Freiheit, 68. Tillich setzt sich in Ders., Die religionsgeschichtliche Konstruktion in Schellings positiver Philosophie, ihre Voraussetzungen und Prinzipien, Breslau 1910, damit auseinander.

${ }_{18}$ Paul Tillich, Systematische Theologie, Bd. I/II (Berlin 1958), Berlin/New York ${ }^{8} 1984$, unveränderter Nachdruck 1987; Ders., Systematische Theologie, Bd. III, Berlin 1966, unveränderter Nachdruck 1987.

19 Paul Tillich, Das System der Wissenschaften nach Gegenständen und Methoden [1923], in: Frühe Schriften (GW I), Stuttgart ${ }^{2} 1959,109-293$.
} 
Weisen des Zusammenhangs der "Urelemente« Denken und Sein ${ }^{20}$ getroffenen Unterscheidung von Geisteswissenschaften, Denkwissenschaften und Seinswissenschaften die unterschiedlichen Disziplinen, die sich in einem Klima wissenschaftlicher Grundsatzdebatten in dieser Zeit methodisch neu aufstellen. ${ }^{21}$ Tillich bedenkt in seiner Schrift alle ihm bekannten Disziplinen und ordnet dabei u.a. die Psychologie den Seinswissenschaften zu, grenzt sie damit von den Geisteswissenschaften $\mathrm{ab}$ und positioniert sich damit hinsichtlich der umstrittenen Frage nach dem Standort jener Wissenschaft. Psychologie gilt ihm zusammen mit der Biologie als Gestaltwissenschaft, einer Untergruppe der Seinswissenschaften. Gestaltwissenschaften schaffen es Tillich zufolge in besonderer Weise, dem Sein auf dem Grund zu kommen, denn es stellt eine große Herausforderung dar, sich ihm anzunähern: Seinswissenschaften sind regelrecht vom "Kampf " von Denken und Sein bestimmt, da sich Letzteres als das Andere gegen seine Erfassung stets verwehrt:

"Die Spannung von Denken und Sein erfüllt jede seinswissenschaftliche Erkenntnis. Die Spannung von Denken und Sein trägt das ganze System. Das `Andere aber, das der Einheit des Denkens widerstrebt, ist die Mannigfaltigkeit des Individuellen. Das Denken will die Einheit, es schafft das Allgemeine, Übergreifende, den Zusammenhang. Das Sein aber stellt sich ihm entgegen als das Besondere, Unfaßbare, Einzelne, das ins Unendliche vom Denken nicht aufzulösen ist. $\mathbb{1}^{22}$

Diese beiden Disziplinen arbeiteten nun mit der Gestalt und nicht etwa mit dem Gesetz wie "physikalische "Seinswissenschaften, und damit mit einer Größe, die durch das Individuelle bestimmt sei und zugleich für das Allgemeine stehe, ${ }^{23}$ die ein geschlossenes Ganzes und doch Glied in einer Kette und von einem inneren Zweck bestimmt sei. ${ }^{24}$ Dieses Beispiel aus Tillichs Wissenschaftstheorie illustriert, wie er versucht, das Wesen der einzelnen Disziplinen als Wissenschaften zu begreifen, sie in ihrer Beziehung zum Denken und Sein zu er-

\footnotetext{
${ }^{20}$ Vgl. Tillich, System, $118 \mathrm{f}$.

${ }^{21} \mathrm{Zu}$ den "Galilei-Konflikten«, mit denen sich Tillich Zeit seines Lebens auseinandersetzen musste, vgl. Werner Schüßler, Naturwissenschaft - Philosophie - Theologie. Paul Tillich zum Problem der sog. "Galilei- Konflikte«, in: Christian Danz u.a. (Hg.), Theology and Natural Science (International Yearbook for Tillich Research/Internationales Jahrbuch für die Tillich-Forschung/Annales internationales de recherches sur Tillich), Bd. 7, Berlin 2012, 45-78.

22 Tillich, System, 135 (Hervorhebung im Original).

${ }^{23}$ Vgl. ebd., 137.

${ }^{24}$ Vgl. ebd., 139.
} 
fassen, um darzulegen, wie sie vom »Prinzip Wissen« bestimmt sind und auf die Wahrheit eines Ganzen blicken. ${ }^{25}$ Wenn Tillich in seiner Systematischen Theologie Jahrzehnte später davon spricht, dass die Einzelwissenschaften das "Material« bereithielten, das die Situation der Menschen erfasse, und damit die Frage nach dessen Existenz implizierten ${ }^{26}$ auf die die christliche Botschaft mit ihren Symbolen antworten wolle, schließt sich der Kreis eines Durchdenkens von disziplinübergreifendem Arbeiten, das bereits dort begann. Der Gefahr des Geisteswissenschaftlers, sich naturwissenschaftlicher Forschung anzubiedern oder sich aufs Geratewohl auf einen populären neurowissenschaftlichen Ansatz zu stürzen, der möglicherweise im eigenen Diskurs hoch umstritten ist, ${ }^{27}$ entgeht er damit nicht nur Tillich kann die Zusammenarbeit tatsächlich begründen und wurde deshalb auch von Forschern anderer Disziplinen als gleichwertiger Gesprächspartner akzeptiert und konnte so u.a. mit Rollo May einen post-freudianischen Existenz-Psychologen zu einer komplexen Angstmonographie bewegen, auf die sein Der Mut zum Sein wiederum reagiert. ${ }^{28}$ Sein System zeigt eindrücklich, dass er reflektiert hat, dass es sich um unterschiedliche Disziplinen mit unterschiedlichen Zugängen und Fundamenten zur Erfassung derWirklichkeit handelt. Die in transdisziplinärer Forschung drängende Aufgabe, die Weltbilder, mit denen Wissenschaften notwendig arbeiten (materialistische, physikalistische, konstruktivitische etc.), stets mit zu reflektieren, ${ }^{29}$ ist bei Tillich erfüllt. Er weiß um die Notwendigkeit der Trennung der Disziplinen, die auch mit einer jeweils spezifischen Methodik einhergeht, die nicht vorschnell von anderen übernommen werden sollte. Bereits 1923 kritisiert Tillich den "Imperialismus der Methoden « ${ }^{30}$, der den Gegenständen selbst nicht mehr gerecht werde. ${ }^{31}$ Er weiß aber ebenso um den Zusammenhang der Disziplinen: Letztlich gibt es eine Einheit, auf die die Disziplinen schauen und ebenso gibt es eine Einheit im Menschen selbst und eine ursprüngliche Einheit mit der ihn umschließenden Wirklichkeit, die dem Theologen zufolge Gottes Wirklichkeit ist. Eine methodisch sinnvolle Trennung in

\footnotetext{
${ }^{25}$ Vgl. ebd., 117.

${ }^{26}$ Vgl. Tillich, Systematische Theologie, Bd. 1, 76f.

${ }^{27}$ Vgl. zur Gefahr der zu schnellen »Anschlussfähigkeit"Jan Plamper, Geschichte und Gefühl. Grundlagen der Emotionsgeschichte, München 2012, 18.

${ }^{28}$ Vgl. Rollo May, Paulus. Reminiscences of a Friendship, London 1974, 23. Tillich antwortet auf: Rollo May, The Meaning of Anxiety (New York 1950), Mansfield Centre 2011.

${ }^{29}$ Vgl. Plamper, Geschichte, 13-17.

30 Tillich, System, 140.

31 Vgl. ebd., 136.
} 
Einzeldisziplinen mit spezifischer Methodik darf sich nicht zu einer Aufsplitterung dieser Wirklichkeit entwickeln, die eine ist. ${ }^{32}$

Tillich arbeitet wirklich transdisziplinär, wobei das "trans" bei Tillich in einem besonderen Sinne als adäquat erscheint, da er in seinem System Aussagen über eine Wirklichkeit trifft, die in einem transzendenten Horizont steht.

\section{Angst}

Angst ist eine zentrale Kategorie in Tillichs System, da sie die mit diesem System beschriebene Wirklichkeit - den Menschen, die Beziehungen, in denen er steht, seine gesamte Welt - spürbar erfasst. Die Möglichkeit des Angsterlebens steht dabei in Wechselwirkung mit Tillichs System selbst, das mit einer Ontologie als seiner Methode arbeitet: $:^{33}$ Für ihn wird die Wirklichkeit bestimmt durch eine Dialektik von Sein und Nichtsein, die letztlich aufgehoben ist im Sein-Selbst. Dass die Wirklichkeit beschrieben und dass überhaupt wahre Aussagen ${ }^{34}$ getroffen werden können, liegt ihm zufolge an dem diese Realität wahrnehmenden Menschen. Tillich will kein System etablieren, das die Welt unabhängig von dem Menschen, der diese erfährt, beschreiben will, weil dies ihm gar zufolge nicht möglich ist: ${ }^{35}$ Es ist der Mensch, der die Wirklichkeit, die Gottes Wirklichkeit ist, wahrnimmt und zwar durch seine Angst. Damit wird sie selbst zu einer »ontologische[n] Qualität (36 $^{36}$.

Tillich spricht von existentiellen Ängsten, die dem Menschen Bedrohungen aufzeigen und die von ganzen Gesellschaften wahrgenommen werden können. ${ }^{37}$ Dabei kann das Erleben von Angst ein dramatisches Erleben und einen extremen Zustand bedeuten, der den Menschen aus seiner Umwelt reißen kann, so dass seine Welt

\footnotetext{
${ }^{32}$ Vgl. zur bleibenden Berechtigung der Unabhängigkeit der Disziplinen bei Tillich auch Werner Schüßler, Naturwissenschaft, 75-78.

${ }_{33}$ Vgl. Tillich, Systematische Theologie, Bd. 1, 193-198.

${ }^{34} \mathrm{Zu}$ seinem Wahrheitsbegriff vgl. ebd., 121-127.

35 "[...] die Existentialphilosophie wollte keine Anthropologie, sie wollte eine Ontologie, aber sie spürte, dass sie nur einen einzigen Zugang zum Sein hatte, den Menschen. So erhielt die Ontologie den Charakter einer Lehre vom Menschen."Paul Tillich, Die Lehre vom Menschen (Union Theological Seminary, New York 1934/35), in: Ders., Frühe Vorlesungen im Exil (1934-1935), Bd. 17, Berlin/Boston 2012, 158188, hier 181.

${ }^{36}$ Tillich, Systematische Theologie, Bd. 1, 224.

${ }^{37} \mathrm{Vgl}$. Tillich, Mut, 48-52.
} 
regelrecht zerbricht. ${ }^{38}$ Das ist kein angenehmer Zustand, denn in ihm erfährt der Mensch das, was Tillich als "Nichtsein« bezeichnet. Der Mensch erlebt die Größe "Nichtsein", die in Jahrhunderten ontologischer Tradition und in der Gestaltung von Heideggers Philosophie Tillich zufolge neu zur Geltung gebracht wurde, in der Weise, dass er mit seiner eigenen Endlichkeit konfrontiert wird. ${ }^{39}$ Aber im Zustand der radikalen Angst selbst ist keine Reflexion von Sein und Nichtsein, kein tiefergehendes Memento mori möglich - es ist vielmehr eine vernichtende, unbegreifliche Situation, so dass Tillich, Kierkegaard und Schelling sie als "Schwindel " beschrieben. ${ }^{40}$ Angst kann den Menschen vor einen Abgrund führen, vor dem er nicht fliehen, noch gegen die Bedrohung kämpfen kann. Dieser Mangel eines adäquaten Umgangs mit der Angst ist ein Kriterium, das deshalb eine Unterscheidung von Angst und Furcht sinnvoll erscheinen lässt, wie Tillich sie deutlich akzentuiert. ${ }^{41}$ Die Differenzierung war zwar schon immer umstritten, doch die Gründe für eine solche werden gegenwärtig z.T. auch von biologischer oder neurobiologischer Wissenschaft von Neuem durchdacht. ${ }^{42}$ Tillich trägt die Unterscheidung mit, weil sie ihm aus der existenzialistischen Tradition einleuchtet, doch er weiß auch, dass eine absolute Trennung weder der Angst noch der Furcht gerecht werden würde und so betont er, dass beide "die gleiche ontologische Wurzel ${ }^{43}$ hätten, doch unterschiedlich in ihrer Aktualität seien. ${ }^{44}$ Hiermit zeigt sich eine dynamische und zugleich realistische Verhältnisbeziehung: Es gibt eine Verwandtschaft, da die ontologische Größe Angst sich wandeln kann. Sie kann zu Furcht werden, damit man mit ihr umgehen und sich der Mensch der Extremsituation, dem kaum zu

\footnotetext{
${ }^{38}$ Es ist die "nackte Angst", die kaum auszuhalten sei, vgl. Tillich, Mut, 37.Von der Radikalität der Angst spricht auch Stefano Micali, Angst als Erschütterung. Metaphysische und methodologische Ausführungen zur Angst: Eine Heidegger-Kritik, in: Ders./Thomas Fuchs (Hg.), Angst. Philosophische, psychopathologische und psychoanalytische Zugänge, Bd. 6, Freiburg/München 22017, 28-55, hier 44-48, Micali rekurriert hier besonders auf die Angsttheorie des Neurologen Kurt Goldstein, mit dem Tillich im engen Austausch stand.

${ }^{39} \mathrm{Vgl}$. Tillich, Mut, 33-35 u.a.

${ }^{40}$ Vgl. Micali, Angst, 44. Damit werden sie Micali zufolge dem Erleben der Angst auch gerecht, anders als Heidegger, der die Angst aufgrund seiner Betonung ihrer offenbarenden Funktion zu so einer abstrakten Größe werden lasse, die nichts mehr mit der erlebten Angst zu tun habe.Vgl. Micali, Angst, 40-45.

${ }^{41}$ Vgl. Tillich, Mut, 35-37.

${ }^{42}$ Vgl. Günther Tembrock, Angst. Naturgeschichte eines psychobiologischen Phänomens, Darmstadt 2000, 9-15; vgl. LeDoux, Angst, 31-33 u.a.

${ }^{43}$ Tillich, Mut, 35.

${ }^{44}$ Vgl. ebd.
} 
ertragenden Schwindel, entziehen kann. Angst, die in einzelnen Momenten mit dem Nichtsein, das real vorhanden ist, konfrontieren kann, ermöglicht dann Partizipation. ${ }^{45}$ Sie erweist sich somit als konstruktive Größe: Angst erschließt die Wirklichkeit, wie sie ist, weist aber zugleich selbst einen Weg, um sie auszuhalten. ${ }^{46}$ Die offenbarende Funktion behält Angst daher selbst noch in ihren deformierten, d.h. in ihren pathologischen Ausprägungen, womit Tillich nicht die destruktive Macht behandlungsbedürftiger Ängste relativiert, jedoch zeigt, dass sie selbst in ihrer verzerrtesten Form auf reale Konflikte hindeutet, die real bedrohen. ${ }^{47}$ Es ist gut, dass Angst sich selbst in dieser Form bemerkbar macht. Auch im neueren Diskurs über die Angst, im Zuge dessen das gefühlte Leben des Menschen insgesamt aufgewertet wird, wird dies betont: Angst erscheint somit als »Indikator« für Beziehungsgeflechte und pathologische Ängste werden zum Ausdruck für gestörte Beziehungswirklichkeiten im individuellen oder gesellschaftlichen Bereich erklärt, die letztlich behandelt und in eine lebensförderliche Richtung überführt werden müssen. ${ }^{48}$ In Tillichs ontologischer Denk- und Sprachwelt wird dies dahingehend ausgedrückt, dass die Angst in Form einer Pathologie einer Bedrohung, letztlich der des immer im Hintergrund stehenden Nichtseins auszuweichen sucht, sie dies aber in einer Weise tut, in der das Ganze der Wirklichkeit aus dem Blick gerät. ${ }^{49}$ So jedoch "reduziert" der Mensch sein Selbst, sich selbst als ganzer Mensch: ${ }^{50}$ "Die Neurose ist der Weg, dem Nichtsein auszuweichen, indem man dem Sein ausweicht. ${ }^{51}$

Emotionen gelten im gegenwärtigen Diskurs als komplexe Konstrukte mit - je nach Emotionstheorie - u.a. kognitiven und voluntativen Elementen..$^{52}$ Sie gelten als Größen, mit denen wir Welt erschließen und das eigene Leben und das Zusammenleben mit Anderen konstituieren. Ethisches Handeln wäre einigen Emotionsforschern zufolge nicht denkbar ohne Emotionen, denn ob etwas richtig oder falsch ist, wird auch gefühlt und dadurch für den

\footnotetext{
${ }^{45}$ Vgl. ebd., 36f.

${ }^{46}$ Vgl. ebd., 37.

${ }^{47}$ Vgl. ebd., $53 f$.

${ }^{48}$ Die "Indikator«-Funktion herausgearbeitet hat Angelika Ebrecht-Laermann, vgl. Dies.,Angst, Bd. 9, Gießen 2014, u.a. 16. Pathologische Angst ist ihr zufolge Ausdruck gescheiterte Symbolisierungen, vgl. ebd., $109 \mathrm{f}$.

${ }^{49} \mathrm{Vgl}$. Tillich, Mut, 54-56.

${ }^{50}$ Vgl. ebd., 54.

${ }^{51}$ Ebd. (Hervorhebung im Original).

${ }^{52}$ Vgl. Sabine A. Döring, Gefühl und Vernunft, in: Spektrum der Wissenschaft: Das Ende der Zeit 5 (2011), 64-67, hier 66 o. Hartmann, Gefühle, 25.
} 
Fühlenden real. ${ }^{53}$ Angst wird im Horizont des neueren Diskurses mitaufgewertet, doch wird sie in ihm ganz unterschiedlich verortet, so dass nicht nur die Frage nach der Unterscheidung von Angst und Furcht umstritten bleibt, sondern ebenso, ob sich diese Emotion, die in besonderer Weise den Menschen zum Nachdenken über sich selbst veranlasst, eine besondere Qualität hat. Tillich bejaht dies. Angst ermöglicht eine Transzendenzwahrnehmung, das Wahrnehmen des dialektischen Seins der Wirklichkeit, die Angst in ihrem Bewusstmachen des Nichtseins aufdeckt. ${ }^{54}$

\section{Wirklich theologisch}

Die Reflexion über Angst, die plötzlich und schonungslos mit realem Nichtsein konfrontieren kann, lässt Tillich zufolge Rückschlüsse auf die Wirklichkeit zu. In diesem Zusammenhang muss in aller Kürze auf die Grundzüge seines theologischen Systems eingegangen werden: Tillich bedient sich philosophischer Traditionen, um mit einer Ontologie die Wirklichkeit zu erschließen. Er beschreibt dabei den Menschen als Schlüssel zurWirklichkeitsbeschreibung. ${ }^{55} \mathrm{In}$ ihm wird also erfahren, was ist. Diese Einsicht teilt er mit Heidegger, der über das Erleben des Menschen, von seinem Sein als Dasein vom Sein sprach. ${ }^{56}$ Die Erfahrung des Menschen in sein System miteinzubeziehen führt nun bei Tillich nicht zu einem Subjektivismus - im Gegenteil führen ihn gerade die Konstruktion der Erfahrung des Menschen zur Begründung der Subjekt-Objekt-Einheit der Wirklichkeit und ihrer Gestaltung als Welt.

Tillich zufolge macht Angsterleben dem Menschen bewusst, dass Sein und Nichtsein, in denen er sich Zeit seines Lebens bewegt, deren Kampf ihn auf seine eigenen Abgründe und den Abgrund des Nichtseins blicken lässt, dialektisch aufgehoben sein müssen im "Sein-Selbst $«{ }^{57}$ Hier kann Schellings Einfluss betont werden. Dieser hatte in seiner Spätzeit einen dynamischen Gottesbegriff entwickelt, in dem Gott nur als lebendig verstanden werden kann, wenn es auch in ihm den "Widerspruch" gibt und er unterschieden wird

\footnotetext{
${ }^{53}$ S. dazu u.a. die Konzeptionen von Martha Nussbaum, Upheavals of Thought. The Intelligence of Emotions, Cambridge 2001 oder Christoph Ammann, Emotionen Seismographen der Bedeutung. Ihre Relevanz für eine christliche Ethik, Stuttgart 2007. Ammann zeigt das am Beispiel Reue, vgl. Ammann, Emotionen, 25-45.

${ }^{54}$ Vgl. Ihben-Bahl, Angst, bes. 258-261.

${ }^{55}$ Vgl. Tillich, Systematische Theologie, Bd. 1, 76f.

${ }^{56}$ Vgl. Martin Heidegger, Sein und Zeit [1927], Tübingen ${ }^{19} 2006,7.13$ u.a.

${ }^{57} \mathrm{Vgl}$. Tillich, Systematische Theologie, Bd. 1, 222-224. $240 \mathrm{f}$.
} 
von dem "was in Gott selbst nicht Er Selbst ist, d.h. in dem, was Grund seiner Existenz ist. " $^{58}$ Keine »Natur Gottes« anzunehmen, keinen irrationalen Grund, keine "Selbstentgegensetzung", die zu einem »lebendige[n] Prozeß der Selbstsetzung $\${ }^{\$ 9}$ führe, ließe ihn zu einem starren Gott werden, der dann auch nichts mit unserer Welt zu tun haben könnte - oder mit uns, die wir auch den Widerspruch notwendig in uns tragen, den er letztlich umschließt. ${ }^{60}$ Tillich, der diesen Gottesbegriff teilt, konzipiert nun eine Ontologie als Theologie, die die Erfahrungen des Menschen und ihn selbst letztlich erklärbar machen soll. Anthropologische Ontologie als Theologie heißt aber auch, dass er nicht bei der Reflexion über das Sein bzw. das Nichtsein "als solches" stehen bleiben kann - Theologie frage letztlich nach dem »Sinn des Seins für uns. ${ }^{61}$ Diesem will Tillich in seiner "Korrelationsmethode« näherkommen: ${ }^{62}$ In Korrelation steht hierbei der Mensch, der sich seiner Endlichkeit bewusst ist, nach ihr fragt und auch eine Antwort auf die Endlichkeit erhält: Unendlichkeit. Denn warum sollte der Mensch mit seiner Endlichkeit hadern? Tillich zufolge deshalb, weil er das Unendliche wahrnimmt. ${ }^{63}$ Auch fühlt sich der Mensch "entfremdet" von sich selbst, dem Anderen, der Gesellschaft, was wiederum darauf verweise, dass er spürt, dass er ursprünglich in eine Einheit mit dem gehört, von dem er sich in seiner Wirklichkeit getrennt weiß - und zwar in die Einheit mit dem Sein-Selbst. ${ }^{64}$

Angst lässt diese Situation, die Endlichkeit und Entfremdung mit ihrer Konsequenz - Sünde - wahrnehmen ${ }^{65}$ und dadurch offenbart sich zugleich, dass der Mensch einen Eindruck von Unendlichkeit und vom nicht-entfremdeten Sein hat. Für Tillich korrelieren die Fragen des Menschen mit der Antwort, auf die die christliche Tradi-

\footnotetext{
${ }^{58}$ Schelling, Freiheit, 71 (Hervorhebung im Original). Vgl. auch Tillich, Schelling, 174.

59 Tillich, Schelling, 175.

${ }^{60}$ Vgl. ebd., 174f. »Das ist aber der irrationale Wille, das Prinzip der Subjektivität, dessen Widerspruch gegen das Prinzip der Objektivität, den Willen der Liebe, die Bedingung von Einzelheit, Selbstheit und Kreatur ist. Denn durch den Widerspruch kommt der Weltprozeß in Fluß, dessen Aufgabe Versöhnung des Widersprechenden ist." ebd., 174.Vgl. auch Schelling, Freiheit, 71.

${ }^{61}$ Tillich, Systematische Theologie, Bd. 1, 30.

${ }^{62}$ Vgl. ebd., 73-80.

${ }^{63}$ Vgl. u.a. ebd., 240f. u. Bd. 2, 14.

${ }^{64}$ Vgl.Tillich, Systematische Theologie, Bd. 1, 75f.

${ }^{65}$ Vgl. Tillich, Systematische Theologie, Bd. 2, 52-55. Auf Angst in Tillichs Hamartiologie, die im zweiten Band seiner Systematischen Theologie entwickelt wird, kann nicht weiter eingegangen werden.
} 
tion mit ihren Symbolen antworten will ${ }^{66}$ denn: Es gibt eine Antwort und sie ist es auch, die - im Regelfall - Angst immer wieder aufhören lässt: Dass der Mensch trotz seines Bewusstseins der realen Bedrohung durch das Nichtsein mit der Konsequenz der Entfremdung "weitermacht", weiterlebt und weiterwirkt, liegt Tillich zufolge in etwas begründet, dass er »Mut zum Sein" nennt. ${ }^{67}$ Seine Quelle ist Gott und zwar der "Gott über Gott«, der jedes theistische Gottesbild übersteigt, ja alles übersteigt, da er letztlich alles ist. ${ }^{68}$

Tillich "bedient" sich der ontologischen Tradition und etabliert trotz aller (post-)kantischen Kritik eine geschlossene Wirklichkeitskonzeption. Die somit beschriebene Wirklichkeit ist die Wirklichkeit Gottes. Dies macht Tillichs Angsttheorie zu einer wirklich theologischen. Diese Theologie beschreibt einen Gott, in dessen Wirklichkeit ein Mensch steht, dem das Instrumentarium gegeben ist, das, was ihn bedroht, aber auch das, was ihn sein lässt, wahrzunehmen. Sie beschreibt einen Gott, der tragenden Mut schenkt und den Menschen in der Welt, die selbst Teil von Gottes Wirklichkeit ist, wirken lässt. Auch dies wird wahrgenommen, denn auch Muterfahrungen werden gefühlt: Freude ist die entsprechende Emotion. ${ }^{69}$

\section{Wirklich hilfreich}

Tillich, der bereits früh transdisziplinär Angst reflektiert und versucht hat, sie in seine ontologisch begründete Theologie zu überführen, bietet mit seinem System eine Grundlage, auf das auch gegenwärtige Angsttheorien aufbauen können, denn das Dasein und Sosein von Angst in ihren unterschiedlichen Ausprägungen wird bei ihm plausibel. In gegenwärtigen Emotionstheorien wird oft betont, dass Emotionen und auf genuine Weise die Angst in irgendeiner Form auf Wirklichkeit verweisen, diese repräsentieren, Wahres über Mensch und Welt aussagen. Diese Behauptungen bleiben aber bloße Behauptungen, wenn sie nicht innerhalb eines Systems begründet werden. ${ }^{70}$ Erst dann wird auch das Zusammenwirken unterschiedlicher Disziplinen verständlich, denn sie repräsentieren die Perspektiven auf

\footnotetext{
${ }^{66}$ Vgl. Tillich, Systematische Theologie, Bd. 1, 76.

${ }^{67} \mathrm{Zu}$ den unterschiedlichen, fragmentarischen Formen dieses Muts, und dem letztlich allein tragenden, im Glauben erlebten Mut vgl. Tillich, Mut, 66-121.

${ }^{68}$ Vgl. ebd., 124-129.

${ }^{69}$ Vgl. ebd., 23.

${ }^{70}$ Dieses deutet auch Sabine A. Döring an: Man bräuchte eine Ontologie und Epistemologie, wenn man Emotionen u.a. axiologische Eigenschaften zugestehe.Vgl. Dies., Allgemeine Einleitung, 49.
} 
die Wirklichkeit und ihre unterschiedlichen Forschungsergebnisse erhellen die Grundfunktion der Angst - und zwar die, Wirklichkeit wahrzunehmen.

Gerade weil Tillich fachfremde Forschungsergebnisse schon immer mit in sein System einbezogen hat, sie das notwendige "Material « sind, das den Menschen und seine Welt erfasst, wird er der Angst und ihrem Facettenreichtum gerecht.

\section{Gottes Mut und Tillichs Beitrag}

Die Auseinandersetzung mit Gefühlen und dezidiert mit Angst ist gefordert. Doch, so notwendig es auch erscheint, sich im disziplinübergreifenden Gespräch erneut auf diese zu stürzen, um den Facettenreichtum von Angst als besonderer Emotion, die sie zweifelsohne ist, zu erweitern, bedarf es eines Systems, das dieses wertvolle Erkenntnisinstrument des Menschen verständlich macht. ${ }^{71}$ Dies bietet Tillich und dies ist sein bleibender Beitrag für eine Theorie der Angst.

Dass eine Theorie der Angst auch zu demjenigen führt, der trotz der Bedrohung durch das Nichtsein Mut zum Sein schenkt, ist Tillichs bleibender Beitrag für die Theologie.

- Dr. Sabine Joy Ihben-Bahl war von 2013 bis 2019 Wissenschaftliche Mitarbeiterin am Seminar für Systematische Theologie der Evangelisch-Theologischen Fakultät Münster und ist zurzeit Vikarin in der Evangelisch-reformierten Gemeinde Göttingen. $\mathrm{Zu}$ ihren Forschungsschwerpunkten gehören die Theologie Paul Tillichs und Johannes Calvins, die Emotionsforschung und der (neue) Realismus.

${ }^{71}$ Angst kann man in ihrer Komplexität nur näherkommen, »beseitigen« könne man sie sowieso nicht. In dem Sinne bestimmt Michael Ermann auch den Auftrag der Psychoanalyse. Ders., Angst und Angsterkrankungen in der Theoriegeschichte der Psychoanalyse und heute, in: Angst, hg. v. Anne Springer/Bernhard Janta/Karsten Münch, Gießen 2011, 13-27, hier 26. 